\title{
Erratum to: Pediatric Reference Values for Tibial Trabecular Bone Mineral Density and Bone Geometry Parameters Using Peripheral Quantitative Computed Tomography
}

Inge Roggen ${ }^{1}$ - Mathieu Roelants ${ }^{2} \cdot$ Isabelle Sioen $^{3} \cdot$ Sara Vandewalle $^{4}$.

Stefaan De Henauw ${ }^{3} \cdot$ Stefan Goemaere $^{4} \cdot$ Jean-Marc Kaufman $^{4} \cdot$ Jean De Schepper $^{1,4}$

Published online: 8 August 2015

(C) Springer Science+Business Media New York 2015

Erratum to: Calcif Tissue Int (2015) 96:527-533

DOI 10.1007/s00223-015-9988-2

The original version of this article unfortunately contained an error in tables. The equations for BMC in Tables 2 and 3 were published incorrectly. The corrected Tables 2 and 3 are given below.

The online version of the original article can be found under doi:10. 1007/s00223-015-9988-2.

Jean De Schepper

jean.deschepper@uzbrussel.be

1 Department of Pediatrics, Universitair Ziekenhuis Brussel,

Vrije Universiteit Brussel, Laarbeeklaan 101, 1090 Brussels,

Belgium

2 Laboratory of Anthropogenetics, Vrije Universiteit Brussel, Brussels, Belgium

3 Department of Public Health, Ghent University, Ghent, Belgium

4 Department of Endocrinology, UZ Gent, Ghent, Belgium 
Table 2 Age-dependent values for skewness (L), median (M) and coefficients of variation (S) to calculate reference data for pQCT analyses of the tibia in males

\begin{tabular}{|c|c|c|c|}
\hline & $\mathrm{L}$ & M & $\mathrm{S}$ \\
\hline BMC $4 \%$ & 0 & $61.552+9.021 \times$ age $+0.52 \times$ age $^{2}$ & $0.193-0.01464 \times$ age $+0.0022 \times$ age $^{2}$ \\
\hline CSA $4 \%$ & 0 & $844.1-169.83 \times$ age $+21.507 \times$ age $^{2}-0.63145 \times$ age $^{3}$ & $0.04+0.0212 \times$ age $-0.00085 \times$ age $^{2}$ \\
\hline $\mathrm{PC} 4 \%$ & 0 & $96.4-10 \times$ age $+1.3664 \times$ age $^{2}-0.04172 \times$ age $^{3}$ & $\begin{array}{l}0.07-0.004 \times \text { age }+0.00096 \times \text { age }^{2} \\
\quad-0.00004 \times \text { age }^{3}\end{array}$ \\
\hline TrvBMD $4 \%$ & 0 & $218.5-5.768 \times$ age $+0.436 \times$ age $^{2}$ & $0.15+0.00367 \times$ age $-0.000247 \times$ age $^{2}$ \\
\hline TvBMD $4 \%$ & 0 & $0.79-15.235 \times$ age $+370.847 \times$ age $^{2}$ & $0.1017786 \times$ age \\
\hline BMC $14 \%$ & 0 & $43.014+9.2217 \times$ age $+0.267 \times$ age $^{2}$ & 0.12743 \\
\hline CSA $14 \%$ & 0 & $445.5-79.457 \times$ age $+9.545 \times$ age $^{2}-0.27413 \times$ age $^{3}$ & $0.17-0.0014 \times$ age \\
\hline EC $14 \%$ & 0 & $66-7.2702 \times$ age $+0.833 \times$ age $^{2}-0.02469 \times$ age $^{3}$ & $0.1-0.00074 \times$ age \\
\hline PC $14 \%$ & 0 & $65.2-4.707 \times$ age $+0.656 \times$ age $^{2}-0.01932 \times$ age $^{3}$ & $0.09-0.0006 \times$ age \\
\hline BMC $38 \%$ & 0 & $42.5746+14.85 \times$ age $+0.31415 \times$ age $^{2}$ & 0.113445 \\
\hline CSA $38 \%$ & 0 & $234.7-24.138 \times$ age $+4.28 \times$ age $^{2}-0.1148 \times$ age $^{3}$ & $0.085+0.0149 \times$ age $-0.00088 \times$ age $^{2}$ \\
\hline CTh $38 \%$ & 0 & $-0.27+0.7 \times$ age $-0.042 \times$ age $^{2}+0.00112 \times$ age $^{3}$ & $\begin{array}{l}0.3-0.0473115 \times \text { age }+0.0035 \times \text { age }^{2} \\
\quad-0.000089 \times \text { age }^{3}\end{array}$ \\
\hline CvBMD $38 \%$ & 0 & $1076-14.9 \times$ age $+1.003 \times$ age $^{2}$ & $-0.016+0.0089 \times$ age $-0.00038 \times$ age $^{2}$ \\
\hline EC $38 \%$ & 0 & $46.2-4.41 \times$ age $+0.514 \times$ age $^{2}-0.01478 \times$ age $^{3}$ & $\begin{array}{l}0.2-0.0175 \times \text { age }+0.0016 \times \text { age }^{2} \\
\quad-0.0000542 \times \text { age }^{3}\end{array}$ \\
\hline PC $38 \%$ & 0 & $38.1+2.42345 \times$ age & $0.04+0.0074 \times$ age $-0.00044 \times$ age $^{2}$ \\
\hline
\end{tabular}

$B M C$ bone mineral content, $C S A$ bone cross-sectional area, $C T h$ cortical thickness, $C v B M D$ cortical volumetric bone mineral density, $E C$ endosteal circumference, $P C$ periosteal circumference, $T v B M D$ total volumetric bone mineral density, $\operatorname{Tr} v B M D$ trabecular volumetric bone mineral density

Table 3 Age-dependent values for skewness (L), median (M) and coefficients of variation (S) to calculate reference data for pQCT analyses of the tibia in females

\begin{tabular}{|c|c|c|c|}
\hline & $\mathrm{L}$ & M & $\mathrm{S}$ \\
\hline BMC $4 \%$ & 0 & $200.559-33.358 \times$ age $+4.342 \times$ age $^{2}-0.12 \times$ age $^{3}$ & $0.2404-0.005 \times$ age \\
\hline CSA $4 \%$ & 0 & $570.4-90.0471 \times$ age $+13.95 \times$ age $^{2}-0.425 \times$ age $^{3}$ & $\begin{array}{l}0.02+0.0421 \times \text { age }-0.00336 \times \text { age }^{2} \\
\quad+0.0000785 \times \text { age }^{3}\end{array}$ \\
\hline PC $4 \%$ & 0 & $84.3-6.50035 \times$ age $+0.991 \times$ age $^{2}-0.0302 \times$ age $^{3}$ & $\begin{array}{l}0.01706 \times \text { age }-0.00112 \times \text { age }^{2} \\
\quad+0.0000229 \times \text { age }^{3}\end{array}$ \\
\hline TrvBMD $4 \%$ & 0 & $259-19.72 \times$ age $+1.776 \times$ age $^{2}-0.04286 \times$ age $^{3}$ & $0.124+0.00866 \times$ age $-0.000426 \times$ age $^{2}$ \\
\hline TvBMD $4 \%$ & 0 & $0.61-13.509 \times$ age $+358.101 \times$ age $^{2}$ & $0.0036+0.064 \times$ age \\
\hline BMC $14 \%$ & 0 & $36.2757+11.624 \times$ age & 0.132625 \\
\hline CSA $14 \%$ & 0 & $259.7-28.72 \times$ age $+5.144 \times \operatorname{age}^{2}-0.1639 \times \operatorname{age}^{3}$ & $0.16-0.00069 \times$ age \\
\hline EC $14 \%$ & 0 & $49.3-3.044 \times$ age $+0.491 \times$ age $^{2}-0.016 \times$ age $^{3}$ & $0.07+0.0063 \times$ age $-0.00027 \times$ age $^{2}$ \\
\hline PC $14 \%$ & 0 & $54.1-2.0454 \times$ age $+0.4514 \times$ age $^{2}-0.0151 \times$ age $^{3}$ & $\begin{array}{l}0.03+0.0109 \times \text { age }-0.00084 \times \text { age }^{2} \\
\quad+0.0000199 \times \text { age }^{3}\end{array}$ \\
\hline BMC $38 \%$ & 0 & $51.9747+16.2265 \times$ age & 0.128311 \\
\hline CSA $38 \%$ & 0 & $235.4-17.1861 \times$ age $+3.409 \times$ age $^{2}-0.1065 \times$ age $^{3}$ & $0.14+0.000297 \times$ age \\
\hline CTh $38 \%$ & 0 & $1.1+0.291 \times$ age $-0.00575 \times$ age $^{2}$ & $\begin{array}{l}0.13+0.0094 \times \text { age }-0.00117 \times \text { age }^{2} \\
\quad+0.0000315 \times \text { age }^{3}\end{array}$ \\
\hline CvBMD $38 \%$ & 0 & $\begin{array}{l}1113-39.0554334 \times \text { age }+4.3320264 \times \text { age }^{2} \\
-0.1 \times 122990 \times \text { age }^{3}\end{array}$ & $0.02+0.00299 \times$ age $-0.00016 \times$ age $^{2}$ \\
\hline EC $38 \%$ & 0 & $43.1-2.5227 \times$ age $+0.305 \times$ age $^{2}-0.00913 \times$ age $^{3}$ & $0.14-0.000642 \times$ age \\
\hline PC $38 \%$ & 0 & $52.0-1.1165439 \times$ age $+0.3030540 \times$ age $^{2}-0.0100136 \times$ age $^{3}$ & $0.073+0.000137 \times$ age \\
\hline
\end{tabular}

$B M C$ bone mineral content, $C S A$ bone cross-sectional area, CTh cortical thickness, $C v B M D$ cortical volumetric bone mineral density, EC endosteal circumference, $P C$ periosteal circumference, $T v B M D$ total volumetric bone mineral density, $\operatorname{Tr} v B M D$ trabecular volumetric bone mineral density 